\title{
Multiresistant Salmonella enterica serovar 4,[5],12:i:- in Europe: a new pandemic strain?
}

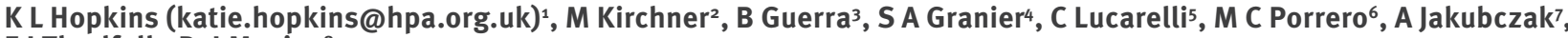

EJ Threlfall ${ }^{1}$, D. J Mevius ${ }^{8,9}$

1. Health Protection Agency Centre for Infections, London, United Kingdom

2. Veterinary Laboratories Agency, Weybridge, United Kingdom

3. Federal Institute for Risk Assessment, Berlin, Germany

4. Agence Française de Sécurité Sanitaire des Aliments, Maisons-Alfort, France

5. Istituto Superiore di Sanitá, Rome, Italy

6. Health Surveillance Centre (VISAVET), University Complutense, Madrid, Spain

7. National Institute of Public Health, Warsaw, Poland

8. Central Veterinary Institute of Wageningen, Lelystad, The Netherlands

9. Faculty of Veterinary Medicine, Utrecht University, The Netherlands

Citation style for this article: Hopkins KL, Kirchner M, Guerra B, Granier SA, Lucarelli C, Porrero MC, Jakubczak A, Threlfall EJ, Mevius DJ. Multiresistant Salmonella enterica serovar 4,[5],12:i:- in Europe: a new pandemic strain?. Euro Surveill. 2010;15(22):pii=19580. Available online: http://www.eurosurveillance.org/ ViewArticle.aspx?Articleld $=19580$

This article has been published on 3 June 2010

A marked increase in the prevalence of $S$. enterica serovar 4,[5],12:i:- with resistance to ampicillin, streptomycin, sulphonamides and tetracyclines (R-type ASSUT) has been noted in food-borne infections and in pigs/pig meat in several European countries in the last ten years. One hundred and sixteen strains of $S$. enterica serovar 4,[5],12:i:- from humans, pigs and pig meat isolated in England and Wales, France, Germany, Italy, Poland, Spain and the Netherlands were further subtyped by phage typing, pulsed-field gel electrophoresis and multilocus variable number tandem repeat analysis to investigate the genetic relationship among strains. PCR was performed to identify the $f l j B$ flagellar gene and the genes encoding resistance to ampicillin, streptomycin, sulphonamides and tetracyclines. Class 1 and 2 integrase genes were also sought. Results indicate that genetically related serovar 4,[5],12:i:- strains of definitive phage types DT193 and DT120 with ampicillin, streptomycin, sulphonamide and tetracycline resistance encoded by bla ${ }_{\mathrm{TEM}}$, strA-strB, sulz and tet(B) have emerged in several European countries, with pigs the likely reservoir of infection. Control measures are urgently needed to reduce spread of infection to humans via the food chain and thereby prevent the possible pandemic spread of serovar 4,[5],12:i:- of R-type ASSuT as occurred with $S$. Typhimurium DT104 during the 1990s.

\section{Introduction}

Infections with Salmonella enterica account for the second largest burden of bacterial gastrointestinal disease in the European Union (EU) [1]. The majority of Salmonella infections result in mild, self-limited illness and may not require treatment with antimicrobials. Nevertheless treatment with an appropriate antimicrobial can be life-saving in immunocompro- mised patients and in invasive disease, such as Salmonella bacteraemia and meningitis.

Serotyping according to the Kauffmann-White scheme is a widely used method for the initial characterisation of Salmonella isolates and is based on the antigenic variability of the somatic $(0)$ and flagellar $(H)$ antigens present in the cell wall of the organism [2]. Despite identification of more than 2,500 different serovars, the majority of cases of human infection are caused by a limited number of serovars. Most serovars are biphasic and express two distinct flagellar antigens encoded by fliC (phase-1 flagellin) and fljB (phase-2 flagellin). However, some serovars fail to express either the phase-1 or phase-2 flagellar antigen, therefore are classed as monophasic.

S. enterica serovar 4,[5],12:i:- is considered a monophasic variant of serovar Typhimurium $(4,[5], 12: i: 1,2)$ due to antigenic and genotypic similarities between the two serovars $[3,4]$. Serovar Typhimurium is the second most common serovar associated with human cases of Salmonella infection in the EU [1]. In contrast isolates of serovar 4,[5],12:i:- were rarely identified before the mid-1990s but are now among the top 10 most common serovars isolated from humans in several countries [3-8]. According to Enter-net data this serovar was the fourth most common serovar in confirmed cases of human salmonellosis in the EU in 2006 [1]. Cases of infection with serovar 4,[5],12:i:- have reportedly been severe, with a $70 \%$ hospitalisation rate during an outbreak in New York City in 1998 [9], although a much lower rate of $21 \%$ was observed during an outbreak in Luxembourg in 2006 [6]. Infections have also been particularly associated with cases of septicaemia in Thailand and Brazil $[7,10]$. Overall, cases of infection have been linked to a number of sources, including 
poultry and cattle, but particularly pigs and pork products [4,6,10-13]. Serovar 4,[5],12:i:- was among the top 10 most common serovars isolated from both pigs and pig meat in the EU in 2006 [1].

A marked increase in prevalence of $S$. enterica serovar 4,[5],12:i:- with resistance to ampicillin, streptomycin, sulphonamides and tetracyclines (R-type ASSuT) has been noted both in food-borne infections and in pigs/pig meat in several European countries over the last ten years $[6,8,14,15]$. In the baseline study from fattening pigs (Commission Decision 2006/668/EC), Spanish strains of S. enterica serovar 4,[5],12:i:- represented $14.3 \%$ of the isolates, $52.5 \%$ of which were of R-type ASSUT (VISAVET Salmonella database, unpublished data). In England and Wales cases of serovar 4,[5],12:i:- infection have risen from 47 in 2005 to 151 in 2009 (a 321\% increase) against a backdrop of an overall decrease in the number of salmonellosis cases, with R-type ASSuT accounting for approximately $30 \%$ of these strains (Health Protection Agency (HPA) Salmonella database, unpublished data). In France isolations of serovar 4,[5],12:i:- increased from 99 to 410 between 2005 and 2008 to become the third most common serovar isolated from humans, with $62 \%$ of strains in 2007 being of R-type ASSuT [16]. In Italy cases of serovar 4,[5],12:i:- infection have risen from 59 in 2003 to 641 in 2009, with $75 \%$ of monophasic strains isolated in 2009 belonging to R-type ASSuT (with or without additional resistances) (Istituto Superiore di Sanità Salmonella database, unpublished data). A recent study described emergence of a clonal group of serovar Typhimurium and 4,[5],12:i:- R-type ASSuT strains in Italy, Denmark and the United Kingdom (UK) [17]. Resistance genes $b l a_{\text {TEM-1 }}$, strA-strB, sul2 and tet(B) encoding resistance to ampicillin, streptomycin, sulphonamides and tetracyclines were localised on the bacterial chromosome. On the basis of resistance gene content and the lack of class 1 integrons these observations have suggested the existence of a new resistance island that differs from the Salmonella Genomic Island-1 [17].

In response to the rapid increase in the frequency of S. enterica serovar 4,[5],12:i:-, R-type ASSuT strains, isolates from England and Wales, Germany, France, Italy, Poland, Spain and the Netherlands were compared using phage typing, resistance gene characterisation, pulsed-field gel electrophoresis (PFGE) and multilocus variable number tandem repeat (MLVA) analysis to evaluate the possibility of clonal spread of this emerging multidrug-resistant (MDR) strain.

\section{Methods and Materials}

\section{Isolate collection}

The eight participating laboratories (the HPA Centre for Infections, London and the Veterinary Laboratories Agency, Weybridge in the UK, the Agence Française de Sécurité Sanitaire des Aliments in Maisons-Alfort, France, the Federal Institute for Risk Assessment in Berlin, Germany, the Istituto Superiore di Sanità in
Rome, Italy, the National Institute of Public Health in Warsaw, Poland, the Health Surveillance Centre (VISAVET), University Complutense in Madrid, Spain and the Central Veterinary Institute of Wageningen in Lelystad, the Netherlands) were asked to submit a maximum of 10 isolates of $S$. enterica serovar 4,[5],12:i:- exhibiting resistance (according to local protocols) to ampicillin, streptomycin, sulphonamides and tetracyclines, and isolated from humans, pigs or pig meat between 2006-2008. In addition, laboratories were invited to send a maximum of 10 isolates of serovar 4,[5],12:i:- exhibiting other resistance phenotypes. All isolates were sent to the HPA.

\section{Strain characterisation}

The Salmonella serotype was confirmed on the basis of the Kauffmann-White scheme and phage typing performed in accordance with HPA protocols $[2,18]$. In addition, isolates were screened using a duplex PCR targeting regions specific to serovar Typhimurium and to definitive phage type (DT) 104 and related strains of phage type (PT) U302 [19]. PCRs targeting the variable regions of the $f l j B$ genes encoding the phase-2 flagellar antigens $H: 1,2, H: 1,5, H: 1,6, H: l, 7, H: e, n, x, H: e, n, Z 15$ and $\mathrm{H}: 1, \mathrm{~W}$, were performed as previously described [20].

Susceptibility to a panel of 18 antimicrobials was determined by a breakpoint method in Isosensitest agar (Oxoid, Basingstoke, UK). The final plate concentrations $(\mu \mathrm{g} / \mathrm{mL})$ used routinely by the HPA on the basis of long-term studies were: ampicillin $(A ; 8)$, chloramphenicol $(C ; 8)$, gentamicin $(G ; 4)$, kanamycin $(K ; 16)$, neomycin ( $\mathrm{Ne} ; 8)$, streptomycin $(S ; 16)$, sulphonamides (Su; 64), tetracycline $(T ; 8)$, trimethoprim (Tm; 2), furazolidone (Fu; 8), nalidixic acid ( $\mathrm{Nx} ; 16)$, ciprofloxacin (low-level (Cpl) 0.125; high-level (Cp) 1.0), amikacin (Ak; 4), cephalexin $(C x ; 16)$, cephradine $(C r ; 16)$, cefuroxime (Cf; 16), ceftriaxone $(\mathrm{Cn} ; 1)$ and cefotaxime $(\mathrm{Ct} ; 1)$. Resistance genes $b_{l} a_{\mathrm{TEM}}, \operatorname{str} A-s t r B$, sul2 and tet(B), and classes 1 and 2 integrase genes were sought by PCR using previous described primers [21,22].

\section{Molecular subtyping}

PFGE was performed after digestion of genomic DNA with Xbal according to a standardised protocol [23]. The patterns were analysed using the Bionumerics software package (version 5.10; Applied Maths, SintMartens-Latem, Belgium) and resulting band profiles were submitted to the PulseNet Europe database for assigning profile names. Dendrograms were constructed using the Dice similarity coefficient and the unweighted pair group method with arithmetic averages (UPGMA) with optimisation and position tolerance set at $1.5 \%$. Multilocus variable number tandem repeat (MLVA) analysis was performed according to a previously described protocol [24]. MLVA profiles were assigned based on the fragment size amplified from each locus, with 'NA' used to denote a locus not present [25]. 


\section{Results}

Some 122 serovar 4,[5],12:i:- isolates were sent to the HPA Laboratory of Gastrointestinal Pathogens, of which 116 were confirmed as serovar 4,[5],12:i:-. These comprised 41 from England and Wales (20 from pigs and 21 from humans, including three from patients with a history of recent travel to Thailand, Greece and an undisclosed destination), 10 isolates from France (isolated from pig meat), 19 from Germany (12 from pigs, six from pig meat and one from a human), 23 from Italy (from humans), five from Poland (from humans), eight from Spain (from pigs) and 10 from the Netherlands (seven from human cases of infection; three from pigs). The $\mathrm{H}: 1,2$ phase-2 flagellar antigen could be serologically detected in the remaining six isolates.

Phage typing using the Typhimurium typing phages identified 16 different PTs (Table 1). The most commonly identified PTs were DT193 (51 isolates), DT120 (27 isolates) and RDNC (reacts but does not conform; 11 isolates). DT193 was the most common PT identified

\section{TABLE 1}

Phage type distribution among serovar 4,[5],12:i:- isolates from seven European countries, 2006-2008 (n=116)

\begin{tabular}{|c|c|}
\hline Country & Phage type (number of isolates) \\
\hline England and Wales & $21 \operatorname{var}(1), 120\left(13^{a}\right), 191(1), 193\left(21^{b}\right), 208$ (1), RDNC (2), U302 (2) \\
\hline France & $68 \operatorname{var}(1), 120(2), 193$ (5), U311 (1), UT (1) \\
\hline Italy & 7 var (3), $18 \operatorname{var}(2), 120(6), 193$ (3), RDNC (5), U311 (3), UT (1) \\
\hline Poland & $120(4), 104(1)$ \\
\hline Spain & 18 (1), 193 (4), RDNC (1), U302 (1), U311 (1) \\
\hline The Netherlands & $12(2), 120(2), 193(6)$ \\
\hline
\end{tabular}

RDNC: isolates that react with the typing phages, but do not conform to a recognised pattern; UT: isolates that do not react with any of the typing phages; var: variant.

Phage type as determined by the scheme of Anderson et al. [18].

a Including two strains associated with foreign travel.

${ }^{b}$ Includes one strain associated with foreign travel.

\section{TABLE 2}

Comparison of phage type and R-type with PFGE profile of serovar 4,[5],12:i:- isolates from seven European countries, 2006-2008 (n=116)

\begin{tabular}{|c|c|c|c|c|c|c|c|c|c|c|c|c|c|c|c|c|c|c|}
\hline \multirow[b]{3}{*}{ PT } & \multicolumn{18}{|c|}{ PFGE profiles (STYMXB.) } \\
\hline & \multicolumn{6}{|c|}{ ASSUT } & \multicolumn{6}{|c|}{ ASSuT and other resistances ${ }^{b}$} & \multicolumn{6}{|c|}{ Other resistance patterns (not ASSuT) ${ }^{c}$} \\
\hline & 0131 & 0083 & 0079 & 0010 & 0022 & Other $^{\mathrm{a}}$ & 0131 & 0083 & 0079 & 0010 & 0022 & Other & 0131 & 0083 & 0079 & 0010 & 0022 & Other \\
\hline 193 & 24 & 1 & & 1 & 2 & 7 & 2 & & & & 1 & 3 & 4 & & 1 & & 1 & 4 \\
\hline 120 & 1 & 4 & 1 & 6 & 1 & 1 & & 4 & 1 & & & 1 & & 2 & 3 & 1 & & 1 \\
\hline RDNC & 1 & & 2 & 1 & & 2 & & & & 1 & & 1 & & & & & & 2 \\
\hline $\mathrm{U}_{311}$ & & & 1 & & 1 & & & & & & & 3 & & & & & & \\
\hline$U_{302}$ & & & 1 & & & & & & & & & 1 & & & & & & 1 \\
\hline 7 var & & & 1 & & & & & & & & & & & & & & & 2 \\
\hline 12 & & & & & & & & & & & & & & & & & & 2 \\
\hline 208 & & & & & & & & & & & & & & & & & & 2 \\
\hline 18 var & & & & & & & & & 1 & & & & & 1 & & & & \\
\hline UT & & & & & & 1 & & & & & & 1 & & & & & & \\
\hline 104 B low & & & & & & & & & & & & & & 2 & & & & \\
\hline 104 low & & & & & & & & & & & & 1 & & & & & & \\
\hline 18 & & & & & & & & & & & & & & & & & & 1 \\
\hline 191 & & & & & & & & & & & & & & & & & & 1 \\
\hline $21 \mathrm{var}$ & & & & & & 1 & & & & & & & & & & & & \\
\hline 68 var & & & & & & & & & & & & & & & & & & 1 \\
\hline Total & 26 & 5 & 6 & 8 & 4 & 14 & 2 & 4 & 2 & 1 & 1 & 11 & 4 & 5 & 4 & 1 & 1 & 17 \\
\hline
\end{tabular}

PFGE: pulsed-field gel electrophoresis.

a Includes two untypable strains.

${ }^{b}$ Includes resistance patterns ACGNeKSSUTTmNxCpl (1 strain), ACKSSuT (1), ACSSuSpTTm (2), AGKNeSSuTTm ( $\left.n=1\right), \operatorname{AGSSpSuT~(n=1),~}$ $\operatorname{AGSSuTTm}(n=1), \operatorname{AKSSuT}(n=1), \operatorname{ASSpSuTNxCpl}(n=1), \operatorname{ASSuTNxCpl}(n=2), \operatorname{ASSuTNxCpl}(n=1)$ and ASSuTTm $(n=9)$.

'Includes fully sensitive strains $(n=6), \operatorname{AGST}(n=1)$, AGSuT $(n=1), \operatorname{AGT}(n=1), \operatorname{ASSu}(n=1)$, ASuT $(n=1)$, SSuTm $(n=1)$, SSuTTm $(n=6)$, SuT (n=1), $\operatorname{SuTTm}(n=1)$ and $T(n=9)$. 
in England and Wales, France, Germany, Spain and the Netherlands, while DT120 predominated in Italy

\section{FIGURE}

Comparison of the five most common PFGE profiles identified in serovar 4,[5],12:i:- isolates from seven European countries, 2006-2008

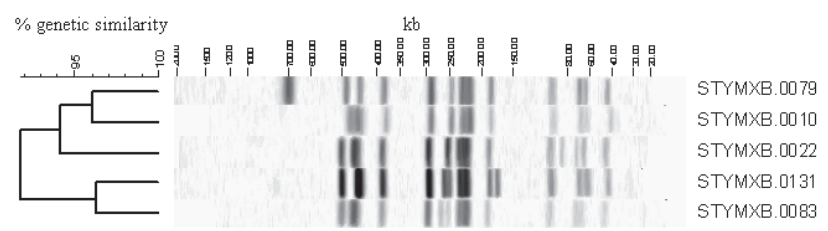

PFGE: pulsed-field gel electrophoresis. and Poland. All 116 isolates were PCR-positive for the Typhimurium-specific fragment of the malic acid dehydrogenase gene but only four isolates (one belonging to DT104, two to PT U302 and one untypable) gave a product with primers targeting the $16 \mathrm{~S}$ to $23 \mathrm{~S}$ spacer region specific to DT104 and the related PT U302 [19].

Overall, 94 of 116 isolates were PCR-negative for all variants of the $f l j B$ gene coding for the phase-2 flagellar antigen, including 48 of 51 DT193 and 17 of 27 DT120 isolates. H:1,2-specific amplicons were detected in the remaining 22 isolates.

Eighty-four isolates (72\%) expressed resistance to ampicillin, streptomycin, sulphonamides and tetracyclines (R-type ASSuT), with or without additional

TABLE 3

Comparison of common PFGE profiles with phage type, country of origin and sources of isolates, 2006-2008 (n=74)

\begin{tabular}{|c|c|c|c|c|}
\hline STYMXB. & Number of isolates & Phage type (number of isolates) & Country of origin (number of isolates.) & Source (number of isolates) \\
\hline 0131 & 32 & $\begin{array}{l}\text { DT193 (30) } \\
\text { DT120 (1) } \\
\text { RDNC (1) }\end{array}$ & $\begin{array}{c}\text { France (2) } \\
\text { The Netherlands (6) } \\
\text { England and Wales (16) } \\
\text { Germany (8) }\end{array}$ & $\begin{array}{c}\text { Humans (10) } \\
\text { Pigs/pig meat (22) }\end{array}$ \\
\hline 0083 & 14 & $\begin{array}{l}\mathrm{DT} 120(10) \\
\mathrm{DT} 104(2) \\
18 \operatorname{var}(1) \\
\mathrm{DT} 193(1) \\
\end{array}$ & $\begin{array}{c}\text { France (2) } \\
\text { England and Wales (9) } \\
\text { Germany (2) } \\
\text { Italy (1) }\end{array}$ & $\begin{array}{r}\text { Humans (5) } \\
\text { Pigs/pig meat (9) }\end{array}$ \\
\hline 0079 & 12 & $\begin{array}{l}\text { DT120 (5) } \\
\text { RDNC (2) } \\
18 \operatorname{var}(1) \\
7 \operatorname{var}(1) \\
\text { U302 (1) } \\
\text { U311 (1) }\end{array}$ & $\begin{array}{l}\text { England and Wales (2) } \\
\qquad \begin{array}{c}\text { Spain (1) } \\
\text { Italy (9) }\end{array}\end{array}$ & $\begin{array}{c}\text { Humans (11) } \\
\text { Pigs/pig meat (1) }\end{array}$ \\
\hline 0010 & 10 & $\begin{array}{l}\text { DT120 (7) } \\
\text { RDNC (2) } \\
\text { DT193 (1) }\end{array}$ & $\begin{array}{c}\text { France (1) } \\
\text { The Netherlands (1) } \\
\text { England and Wales (1) } \\
\text { Poland (3) } \\
\text { Spain (1) } \\
\text { Italy (1) } \\
\text { Germany (2) }\end{array}$ & $\begin{array}{l}\text { Humans (5) } \\
\text { Pigs/pig meat (5) }\end{array}$ \\
\hline 0022 & 6 & $\begin{array}{l}\text { DT193 (4) } \\
\text { DT120 (1) } \\
\text { U311 (1) }\end{array}$ & $\begin{array}{c}\text { France (2) } \\
\text { England and Wales (1) } \\
\text { Poland (1) } \\
\text { Spain (1) } \\
\text { Germany (1) }\end{array}$ & $\begin{array}{c}\text { Humans (2) } \\
\text { Pigs/pig meat (4) }\end{array}$ \\
\hline
\end{tabular}

PFGE: pulsed-field gel electrophoresis. 
resistance(s) (Table 2). Six isolates were fully sensitive to all antimicrobials in the test panel. Eighty-three of 92 ampicillin-resistant iolates carried $b l a_{\text {TEM }}, 85$ of 96 streptomycin-resistant isolates carried strA-strB,

TABLE 4

Subdivision of the five most common PFGE profiles using MLVA analysis, 2006-2008 ( $\mathrm{n}=74)$

\begin{tabular}{|c|c|c|c|c|c|c|c|}
\hline \multirow{3}{*}{ PFGE pattern } & \multirow{3}{*}{ Number of strains } & \multicolumn{6}{|c|}{ MLVA profile (based on number of tandem repeats at each locus) ${ }^{\mathrm{a}}$} \\
\hline & & \multirow{2}{*}{ SSTR9 } & \multirow{2}{*}{ STTR5 } & \multirow{2}{*}{ STTR6 } & \multirow{2}{*}{ STTR10 } & \multicolumn{2}{|c|}{$\mathrm{STRR}_{3}$} \\
\hline & & & & & & 27 bp & $33 \mathrm{bp}$ \\
\hline \multirow{8}{*}{ STYMXB.0131 } & 10 & 3 & 11 & 9 & NA & 2 & 11 \\
\hline & 8 & & 13 & 10 & & & \\
\hline & 5 & & 12 & & & & \\
\hline & 3 & & 13 & & & & \\
\hline & 3 & & & 10 & & & \\
\hline & 1 & & & 12 & & & \\
\hline & 1 & & & 14 & & & \\
\hline & 1 & & & 8 & & & \\
\hline \multirow{7}{*}{ STYMXB.0083 } & 5 & 3 & 12 & 9 & NA & 2 & 11 \\
\hline & 4 & & & 6 & & & \\
\hline & 1 & & 11 & 11 & & & \\
\hline & 1 & & 11 & 12 & & & \\
\hline & 1 & & 13 & 10 & & & \\
\hline & 1 & & 14 & & & & \\
\hline & 1 & 13 & 13 & NA & & & \\
\hline \multirow{9}{*}{ STYMXB.0079 } & 2 & 3 & 12 & 11 & NA & 2 & 11 \\
\hline & 2 & & 13 & 10 & & & \\
\hline & 1 & & 11 & 8 & & & \\
\hline & 1 & & 12 & 12 & & & \\
\hline & 1 & & 12 & 9 & & & \\
\hline & 1 & & 13 & 12 & & & \\
\hline & 1 & & 13 & 9 & & & \\
\hline & 2 & 11 & 15 & NA & & & \\
\hline & 1 & 13 & 13 & NA & & & \\
\hline \multirow{7}{*}{ STYMXB.0010 } & 3 & 3 & 14 & 9 & NA & 2 & 11 \\
\hline & 2 & & 12 & 10 & & & \\
\hline & 1 & & 12 & 7 & & & \\
\hline & 1 & & 12 & 9 & & & \\
\hline & 1 & & 13 & 7 & & & \\
\hline & 1 & & 14 & 10 & & & \\
\hline & 1 & & 15 & 10 & & & \\
\hline \multirow{5}{*}{ STYMXB.0022 } & 2 & 3 & 12 & 9 & NA & 3 & 11 \\
\hline & 1 & & & 13 & & & \\
\hline & 1 & & 13 & 11 & & & \\
\hline & 1 & & 14 & 9 & & 2 & \\
\hline & 1 & & 15 & 10 & & 2 & \\
\hline
\end{tabular}

NA: locus not present; PFGE: pulsed-field gel electrophoresis; MLVA: multilocus variable number tandem repeat analysis.

a Number of tandem repeats only listed where it differs from the most common repeat number in each PFGE profile. 
88 of 99 sulphonamide-resistant isolates carried sul2 and 93 of 105 tetracycline-resistant isolates carried tet(B) (data not shown). Of 84 R-type ASSuT strains, 68 possessed bla $a_{\text {TЕM }}$, strA-strB, sul 2 and tet(B) resistance genes. Eighty-two percent of RDNC isolates, $80 \%$ of DT193 and $74 \%$ of DT120 were of R-type ASSuT (with/without additional resistance(s)), with resistance encoded by genes bla ${ }_{\text {TEM }}$, strA-strB, sul 2 and tet(B) in $78 \%, 75 \%$ and $56 \%$ of isolates respectively. Isolates of R-type ASSuT were negative for both class 1 and 2 integrase genes; these were found only in strains expressing resistance to aminoglycosides and/or trimethoprim. Among the remaining 16 R-type ASSuT strains from the present study that did not carry bla $a_{\text {TEM }}$, strA-strB, sul2 and tet(B), 11 strains lacked only one of tet(B), bla $a_{\text {TEM-1 }}$ or sul2, one strain each lacked bla $a_{\text {TEM-1 }}$ and tet(B) or strA-strB and tet(B), one strain lacked bla $a_{\mathrm{TEM}-1}$, strA-strB and sul2 and one strain lacked all four genes. These strains belonged to phage types DT120 (five strains), DT193 (four strains), RDNC (two strains), and one each belonged to phage types DT104, DT18 variant, U302, U311 and UT.

PFGE analysis identified 36 unique banding profiles among 114 strains; two strains were untypable. These were grouped into 12 clusters of two or more strains and 23 patterns corresponding to a single isolate (data not shown). Sixty-five percent (74/114) of strains were represented by one of five band-

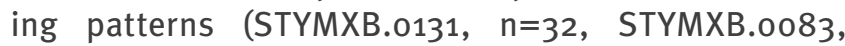
$\mathrm{n}=14$, STYMXB.0079, n=12, STYMXB.0010, $\mathrm{n}=10$, and STYMXB.0022, $\mathrm{n}=6$ ) that shared more than $90 \%$ similarity (Figure, Table 3). Strains from humans and pigs or pig meat were represented in each common PFGE pattern. The majority of strains with PFGE patterns STYMXB.0131 and STYMXB.0022 were phage type DT193, while patterns STYMXB.0083, STYMXB.0079 and STYMXB.0010 were dominated by phage type DT120 (Table 3). Some country-specific differences were noted within the distribution of PFGE patterns: nine of the 12 STYMXB.0079 strains were from Italy, three of the five Polish strains were STYMXB.0010 and six of 10 strains from the Netherlands were pattern STYMXB.0131 (Table 3). STYMXB.0010 was the only profile identified in all seven countries. However, larger numbers of strains need to be analysed to determine whether these country-specific distributions hold true. Patterns STYMXB.0131 and STYMXB.0010 were dominated by R-type ASSuT strains (representing $81 \%$ and $80 \%$ of strains, respectively), whereas resistance profiles of the other common PFGE profiles were more variable (Table 2 ).

MLVA typing identified 45 different profiles that differed by loss or addition of tandem repeats at loci STTR9, STTR5, STTR6 and STTR3, and was able to further subdivide the five most common PFGE profiles (Table 4$)$. Ninety-one percent $(105 / 116)$ of strains failed to amplify a fragment from the Typhimurium-specific virulence plasmid pSLT-bound locus STTR10. The five most common MLVA profiles (3-11-9-NA-211, $n=12$;
3-12-9-NA-211, $n=13 ; 3-13-10-N A-211, n=12 ; 3-14-9-N A-$ 211, $n=7$; and 3-13-9-NA-211, $n=6$ ) accounted for $43 \%$ of strains and differed by only one to three tandem repeats at locus STTR5 and one repeat at locus STTR6.

The most frequently occurring combination of phenotypic and genotypic characteristics was that 51 of 116 (44\%) serovar 4,[5],12:i:- isolates belonged to phage type DT193. Of these 51, 48 were PCR-negative for fljB. Among the 51 DT193 isolates, 37 were of R-type ASSuT (plus additional resistance to chloramphenicol, aminoglycosides and/or trimethoprim in four isolates) encoded by bla $a_{\mathrm{TEM}}$, strA-strB, sul2 and tet(B), and 36 exhibited PFGE profile STYMXB.0131, which could be further divided into eight related MLVA profiles. Isolates bearing these characteristics were isolated in England and Wales (including one isolate from a patient with history of recent travel to Thailand), France, Germany and the Netherlands.

\section{Discussion}

Antimicrobial resistance is a serious public health problem limiting the therapeutic options available to clinicians treating complicated Salmonella infections. In recent years there has been an overall decline in the level of resistance in serovar Typhimurium in several European countries as a result of a reduction in the number of isolates of penta-resistant DT104 [14]. To some extent this reduction has been counteracted by an increase in prevalence of serovar $4,[5], 12$ :i:- isolates expressing resistance to ampicillin, streptomycin, sulphonamides and tetracyclines $[8,17]$.

One of the first reports of serovar 4,[5],12:i:- in Europe was of an isolate grown in the late 1980 srom a chicken carcass in Portugal [26]. This serovar emerged in Spain in strains from humans and pork or pork products during 1997, and subsequently became the fourth most common Salmonella serovar identified from 1998 to 2000 [11]. All isolates belonged to phage type U302. These isolates were classed as monophasic variants of serovar Typhimurium due to presence of an IS2000 fragment located in a Typhimurium-specific location within the fliB-fliA intergenic region and amplification of a Typhimurium DT104- and U302-specific region [3]. All 116 monophasic isolates in this study harboured the Typhimurium-specific fragment of the malic acid dehydrogenase gene, suggesting that these strains are monophasic variants of serovar Typhimurium. However, the majority (97\%) were negative for the DT104- and U302-specific region, suggesting that these monophasic isolates may not be related to the serovar 4,[5],12:i:- strain(s) that emerged in Spain. This was confirmed by phage typing, which identified DT193 as the most common PT, followed by DT120, thereby adding to the diversity of phage types of serovar 4,[5],12:i:linked to serovar Typhimurium. DT193 and DT120 have consistently fallen within the top five phage types of serovar Typhimurium from cases of human infection in England and Wales in recent years (HPA Salmonella database, unpublished data). It is plausible that at 
least some of this increase may be attributed to the emergence of serovar 4,[5],12:i:- DT193 and DT120 strains. Putative Typhimurium isolates sent from primary diagnostic laboratories to the HPA Salmonella Reference Unit are only phage-typed and not routinely subjected to further serological examination. This may result in misclassification as serovar Typhimurium and under-reporting of this serovar in England and Wales, and in other countries where phage typing is used in lieu of full serotyping to identify strains as serovar Typhimurium. Serovar 4,[5],12:i:- DT193 strains have previously been isolated from human cases of infection and/or pigs in Luxembourg and Spain $[6,13]$, while monophasic DT120 strains were identified in Italy [8].

The Spanish PT U302 serovar 4,[5],12:i:- strains were PCR-negative for $\mathrm{H}: 1,2$ [11], as were the majority (81\%) of monophasic isolates in this study. Previous published work has shown that the lack of phase-2 flagellar expression may be due to different mutations (including point mutations) and partial or complete deletions in $f l j B$ and adjacent genes $[4,27]$. Monophasic strains in which the phase-2 flagellar antigen is not detected serologically but can be detected by PCR may contain deletions in a part of $f l j B$ that leave the $\mathrm{H}: 1,2$-specific PCR primer binding sites intact, or they may represent 'serotype inconsistent' strains [27]. These are serovar Typhimurium strains in which serological detection of the phase-2 flagellar antigen may be inconsistent. This may be due to problems with flagellar phase reversal, which is a time-consuming and technically demanding procedure that may result in misclassification of Typhimurium strains as serovar 4,[5],12:i:-. Alternatively, the invertible promoter controlling expression of $\mathrm{fljB}$ and fliC may have become locked in one position allowing only expression of fliC in these strains [4]. The range of mechanisms that can result in non-expression of the phase-2 flagellar antigen make definitive identification of serovar 4,[5],12:i:- problematic. It is possible that molecular serotyping could be used as a basis to define such strains as serovar 4,[5],12:i:- or Typhimurium, but as yet such methods lack standardisation, are not in place in most countries and may not be suitable for laboratories other than reference facilities. Given that there may be discrepancy in detection of the phase-2 flagellar antigen between classical and molecular serotyping, an international agreement both on the definition of monophasic strains and on detection methodology is required. Without reaching such a consensus the true incidence of such Typhimurium-like strains is difficult to assess; only the harmonisation and the sharing of methods will allow accurate comparison of reported data.

In contrast to the monophasic variants isolated in Thailand and Spain, which commonly expressed additional resistance to gentamicin and trimethoprim-sulphamethoxazole and/or chloramphenicol $[10,11]$ and to serovar 4,[5],12:i:- strains isolated in Brazil and New York City, which were infrequently MDR $[7,9]$, the countries participating in this study observed an increase in isolates of serovar 4,[5],12:i:- with resistance to ampicillin, streptomycin, sulphonamides and tetracyclines only. Characterisation of the resistance genes responsible for this phenotype identified $b l a_{\text {TEM }}$, strA$\operatorname{str} B$, sul2 and tet(B) in $81 \%$ of isolates. Such genes have also been identified in isolates of Typhimurium DT193 R-type ASSuT obtained during 2005 in England and Wales from raw beef and a human case of infection, although the majority of strains tested harboured tet(A) rather than tet(B) (unpublished data). Analysis of a $10 \mathrm{~kb}$ chromosomal region of a Typhimurium DT193 revealed the presence of an strB-strA-sul2-repC-repA region derived from plasmid RSF1010 located upstream of bla $a_{\text {TEM-1 }}$ and downstream of a class 1 integron [28]. The resistance genes encoding the tetra-resistant phenotype in isolates of serovars Typhimurium and 4,[5],12:i:- from Italy, Denmark and the UK were also identified as bla $a_{\text {TEM-1 }}$, strA-strB, sul2 and tet(B), but all isolates were negative for class 1 integrons [17]. Transfer experiments were unsuccessful and probes specific for these genes bound to a $750 \mathrm{~kb}$ l-Ceul digest fragment, suggesting a chromosomal location and existence of a new resistance island. As in the present study, strains with other R-types than ASSuT, but with related PFGE profiles and harbouring one or more of bla $a_{\mathrm{TEM}-1}, \operatorname{str} A-s t r B$, sul2 and tet(B) were identified. This suggests that rearrangements or deletions may occur within the resistance island leading to partial resistance patterns [17]. In contrast, resistance to ampicillin, streptomycin, sulphonamides and tetracyclines was mediated by plasmid-borne bla $a_{\mathrm{TEM}-1}$ and tet(A), and a class 1 integron harbouring aadA2 and sul1 in the Spanish serovar 4,[5],12:i:- U302 isolates [29].

Thirty-six profiles were identified among the 114 strains typable by PFGE, thereby supporting previous observations that serovar $4,[5], 12: \mathrm{i}:-$ can demonstrate considerable diversity, even among strains from a single country $[4,9,13,27]$. However, serovar 4,[5],12:i:strains have been reported to be less heterogenic than serovar Typhimurium strains $[9,27,30]$. Serovar Typhimurium demonstrates considerable diversity as evidenced by phage typing and molecular typing, but with certain clonal strains such as multidrug-resistant DT104 [31]. The most common PFGE profile identified in our study was STYMXB.0131, which, together with four other closely related banding patterns (STYMXB.0022, STYMXB.0079, STYMXB.0010 and STYMXB.0083), accounted for $65 \%$ of isolates. Previously submitted STYMXB.0131 patterns in the PulseNet Europe database belonged to serovar Typhimurium DT193 and PT507 (according to the Dutch phage typing scheme) strains isolated from human cases of infection in Finland, the Netherlands and England and Wales. Patterns STYMXB.0131 and STYMXB.0022 have also been identified in Typhimurium DT193 strains from humans, cattle and raw beef in England and Wales (unpublished data), while patterns STYMXB.0083 and STYMXB.0010 have been identified in Typhimurium DT120 isolates in England and Wales and in Denmark [32]. These observations are consistent with previous 
studies that serovar 4,[5],12:i:- strains are genotypically closely related to serovar Typhimurium [4,7,8,27]. Patterns STYMXB.0079 and STYMXB.0010 represented $58 \%$ of serovar Typhimurium R-type ASSuT strains in Italy [8]. Pattern STYMXB.0131 has also been identified among Danish serovar 4,[5],12:i:- strains [17]. Serovar 4,[5],12:i:- R-type ASSuT strains belonging to profile STYMXB.0131 were responsible for two major outbreaks in Luxembourg in 2006 where pork meat was suspected as the vehicle for the outbreaks [6]. In Italy, profiles STYMXB.0079 and STYMXB.0010 represented $83 \%$ of serovar 4,[5],12:i:- R-type ASSuT strains [8]. However, the majority of strains were phage type U302 or untypable; only $8 \%$ of the isolates belonged to DT120 and none were DT193.

MLVA typing was also applied to the strain panel as the technique is reportedly more discriminatory than PFGE and provides unambiguous typing data that is free of the bias generated by differences in resistance genotype that reportedly affects PFGE [33]. Using the nomenclature of Larsson et al. allowed easy recognition of related profiles [25]. The five most common MLVA profiles identified in this study, and single locus variants thereof, have previously been identified in S. Typhimurium DT193 R-type ASSuT strains isolated from humans, pigs, cattle and beef products in England and Wales in 2005-2006 (unpublished data) and in isolates of Typhimurium DT120 R-type ASSuT associated with a putative outbreak in humans in the northeast of England in 2006 [32]. That all monophasic strains were typable by MLVA, using the Lindstedt et al. Typhimurium-specific scheme [24], and shared closely related profiles with these Typhimurium isolates provides tentative further evidence that monophasic 4,[5],12:i:- isolates derive from serovar Typhimurium.

The data presented here suggest that a serovar 4,[5],12:i:- DT193 R-type ASSuT clone with PFGE profile STYMXB.0131 has emerged from serovar Typhimurium and spread within several European countries, with pigs as a likely reservoir of infection. Isolates of serovar 4,[5],12:i:- DT120 R-type ASSuT with closely related PFGE profiles were identified in humans and pigs from five of the participating countries. The diversity of PFGE and MLVA profiles within serovar 4,[5],12:i:- DT193 and DT120 R-type ASSUT isolates, and the differences between these isolates and those previously described in Spain [30], suggests that serovar 4,[5],12:i:- is likely to represent several clones or strains that have emerged independently from serovar Typhimurium. Recent genotypic studies have shown that in addition to the Spanish 4,[5],12:i- clone, other 4,[5],12:i:- lineages exist [27].

In the first ten months of $2009, D_{1} 193$ and DT120 accounted for $18 \%$ and $11 \%$ of Typhimurium isolates in England and Wales, respectively. In contrast, DT104 accounted for only $7 \%$ of Typhimurium isolates (HPA Salmonella database, unpublished data). Serovar 4,[5],12:i:- has already caused substantial outbreaks in several countries, with reports of severe infections and also deaths $[6,7,9,10]$. In order to prevent a global epidemic of these newly emerging clones or strains, as occurred with Typhimurium DT104, appropriate intervention strategies need to be put in place as soon as possible, particularly in pig husbandry throughout the EU.

\section{Acknowledgements}

This study was funded by European Community Network of Excellence MED-VET-NET workpackage 21 (contract No. FOOD-CT-2004-506122). The authors wish to thank Kate Martin and Tansy Peters of the HPA Laboratory of Gastrointestinal Pathogens for technical assistance and submission of PFGE data to the PulseNet Europe database, respectively, and Chris Lane and Ian Fisher for interrogation of the HPA Salmonella database.

\section{References}

1. European Food Safety Authority. The community summary report on trends and sources of zoonoses, zoonotic agents, antimicrobial resistance and foodborne outbreaks in the European Union in 2006. EFSA J. 2007;130:2-352. Available from: http://www.efsa.europa.eu/en/scdocs/scdoc/13or.htm

2. Bale JA, de Pinna EM, Threlfall EJ, Ward LR. Kauffmann-White Scheme - 2007: Salmonella Identification - Serotypes and Antigenic Formulae. London: Health protection Agency; 2007.

3. Echeita MA, Herrera S, Usera MA. Atypical, fljB-negative Salmonella enterica subsp. enterica strain of serovar 4,5,12:i: appears to be a monophasic variant of serovar Typhimurium. J Clin Microbiol. 2001;39(8):2981-3.

4. Zamperini K, Soni V, Waltman D, Sanchez S, Theriault EC, Bray J, et al. Molecular characterization reveals Salmonella enterica serovar 4,[5],12:i:- from poultry is a variant Typhimurium serovar. Avian Dis. 2007;51(4):958-64

5. Switt Al, Soyer Y, Warnick LD, Wiedmann M. Emergence, distribution, and molecular and phenotypic characteristics of Salmonella enterica serotype 4,5,12:i:-. Foodborne Pathog Dis. 2009;6(4):407-15

6. Mossong J, Marques P, Ragimbeau C, Huberty-Krau P, Losch S, Meyer G, et al. Outbreaks of monophasic Salmonella enterica serovar 4,[5],12:i:- in Luxembourg, 2006. Euro Surveill. 2007;12(6). pii=719. Available from: http://www. eurosurveillance.org/ViewArticle.aspx?Articleld=719

7. Tavechio AT, Ghilardi AC, Fernandes SA. "Multiplex PCR" identification of the atypical and monophasic Salmonella enterica subsp. enterica serotype 1,4,[5],12:i:- in São Paulo State, Brazil: frequency and antibiotic resistance patterns. Rev Inst Med Trop Sao Paulo. 2004;46(2):115-7.

8. Dionisi AM, Graziani C, Lucarelli C, Filetici E, Villa L, Owczarek, $S$ et al. Molecular characterization of multidrug-resistant strains of Salmonella enterica serotype Typhimurium and Monophasic variant (S. 4,[5],12:i:-) isolated from human infections in Italy. Foodborne Pathog Dis. 2009;6(6):711-7.

9. Agasan A, Kornblum J, Williams G, Pratt CC, Fleckenstein $\mathrm{P}$, Wong $M$, et al. Profile of Salmonella enterica subsp. enterica (subspecies I) serotype 4,5,12:i:- strains causing food-borne infections in New York City. J Clin Microbiol. 2002;40(6):1924-9.

10. Pornruangwong S, Sriyapai T, Pulsrikarn C, Sawanpanyalert P, Boonmar S, Bangtrakulnonth A. The epidemiological relationship between Salmonella enterica serovar Typhimurium and Salmonella enterica serovar 4,[5],12:i:- isolates from humans and swine in Thailand. Southeast Asian J Trop Med Public Health. 2008;39(2):288-96.

11. Echeita MA, Aladueña A, Cruchaga S, Usera MA. Emergence and spread of an atypical Salmonella enterica subsp. enterica serotype 4,5,12:i:- strain in Spain. J Clin Microbiol. 1999;37(10):3425.

12. Vieira-Pinto $M$, Temudo $P$, Martins C. Occurrence of Salmonella in the ileum, ileocolic lymph nodes, tonsils, mandibular lymph nodes and carcasses of pigs slaughtered for consumption. J Vet Med B Infect Dis Vet Public Health. 2005;52(10):476-81. 
13. de la Torre E, Zapata D, Tello M, Mejía W, Frías N, García Peña FJ, et al. Several Salmonella enterica subsp. enterica serotype 4,5,12:i:- phage types isolated from swine samples originate from serotype typhimurium DT U302. J Clin Microbiol. 2003;41(6):2395-400.

14. Meakins S, Fisher IS, Berghold C, Gerner-Smidt P, Tschäpe $H$, Cormican $M$, et al. Antimicrobial drug resistance in human nontyphoidal Salmonella isolates in Europe 2000-2004: a report from the Enter-net International Surveillance Network. Microb Drug Resist. 2008;14(1):31-5.

15. van Pelt W, Notermans D, Mevius D, Vennema H, Koopmans MPG, van Duynhoven YTHP. Trends in gastro-enteritis van 1996 - 2006: verdere toename van ziekenhuisopnames, maar stabiliserende sterfte. [Further increase in hospital admissions, but stabilising deaths]. Infectieziekten Bulletin. 2008;19(1):24-31. [Dutch].

16. Institut Pasteur. Centre National de Référence des Salmonella - Rapport d'activité annuel 2008. [National Reference Centre for Salmonella - Annual activities report 2008]. Paris: Institut Pasteur; 2009. [French].

17. Lucarelli C, Dionisi AM, Torpdahl M, Villa L, Graziani C, Hopkins $\mathrm{KL}$, et al. Evidence for a second genomic island conferring resistance in a clonal group of Salmonella Typhimurium and its monophasic variant circulating in Italy, Denmark and United Kingdom. J Clin Microbiol. 2010;48(6):2103-9.

18. Anderson ES, Ward LR, Saxe MJ, de Sa JD. Bacteriophagetyping designations of Salmonella typhimurium. J Hyg (Lond). 1977;78(2):297-300.

19. Amavisit $P$, Boonyawiwat $W$, Bangtrakulnont A. Characterization of Salmonella enterica serovar Typhimurium and monophasic Salmonella serovar 1,4,[5],12:i:isolates in Thailand. J Clin Microbiol. 2005;43(6):2736-40.

20. Echeita MA, Herrera S, Garaizar J, Usera MA. Multiplex PCR-based detection and identification of the most common Salmonella second-phase flagellar antigens. Res Microbiol. 2002;153(2):107-13.

21. Hopkins KL, Batchelor MJ, Anjum M, Davies RH, Threlfall EJ. Comparison of antimicrobial resistance genes in nontyphoidal salmonellae of serotypes Enteritidis, Hadar, and Virchow from humans and food-producing animals in England and wales. Microb Drug Resist. 2007;13(4):281-8.

22. Batchelor $M$, Hopkins KL, Liebana E, Slickers $P$, Ehricht R, Mafura M, et al. Development of a miniaturised microarraybased assay for the rapid identification of antimicrobial resistance genes in Gram-negative bacteria. Int J Antimicrob Agents. 2008;31(5):440-51.

23. Peters TM, Maguire C, Threlfall EJ, Fisher IS, Gill N, Gatto AJ, et al. The Salm-gene project - a European collaboration for DNA fingerprinting for food-related salmonellosis. Euro Surveill. 2003;8:(2). pii=401. Available from: http://www. eurosurveillance.org/ViewArticle.aspx?Articleld=401

24. Lindstedt BA, Vardund T, Aas L, Kapperud G. Multiple-locus variable-number tandem-repeats analysis of Salmonella enterica subsp, enterica serovar Typhimurium using PCR multiplexing and multicolor capillary electrophoresis. Microbiol Methods. 2004;59(2):163-72.

25. Larsson JT, Torpdahl M, Petersen RF, Sorensen G, Lindstedt BA, Nielsen EM. Development of a new nomenclature for Salmonella Typhimurium multilocus variable number of tandem repeats analysis (MLVA). Euro Surveill. 2009;14(15). pii:19174. Available from: http://www.eurosurveillance.org/ViewArticle. aspx?Articleld $=19174$

26. Machado J, Bernardo F. Prevalence of Salmonella in chicken carcasses in Portugal. J Appl Bacteriol. 1990;69(4):477-80.

27. Soyer Y, Moreno Switt A, Davis MA, Maurer J, McDonough PL, Schoonmaker-Bopp DJ, et al. Salmonella 4,5,12:i:-: an emerging Salmonella serotype that represents multiple distinct clones. J Clin Microbiol. 2009;47(11):3546-56.

28. Daly M, Villa L, Pezzella C, Fanning S, Carattoli A. Comparison of multidrug resistance gene regions between two geographically unrelated Salmonella serotypes. J Antimicrob Chemother. 2005;55(4):558-561.

29. Guerra B, Soto SM, Argüelles JM, Mendoza MC. Multidrug resistance is mediated by large plasmids carrying a class 1 integron in the emergent Salmonella enterica serotype [4,5,12:i:-]. Antimicrob Agents Chemother. 2001;45(4):1305-8.

30. Guerra B, Laconcha I, Soto SM, Gonzalez-Hevia MA, Mendoza MC. Molecular characterisation of emergent multiresistant Salmonella enterica serotype [4,5,12:i:-] organisms causing human salmonellosis. FEMS Microbiol Lett. 2000;190(2):341-7.

31. Threlfall EJ. Epidemic Salmonella typhimurium DT 104--a truly international multiresistant clone. J Antimicrob Chemother. 2000;46(1):7-10.
32. Best EL, Hampton MD, Ethelberg S, Liebana E, Clifton-Hadley FA, Threlfall EJ. Drug-resistant Salmonella Typhimurium DT 120: use of PFGE and MLVA in a putative international outbreak investigation. Microb Drug Resist. 2009;15(2):133-8.

33. Lindstedt BA, Heir E, Gjernes E, Kapperud G. DNA fingerprinting of Salmonella enterica subsp. enterica serovar Typhimurium with emphasis on phage type DT104 based on variable number of tandem repeat loci. J Clin Microbiol. 2003;41(4):1469-79. 